\title{
The Effect of Medium-Intensity Electromagnetic Stimulation on Pelvic Floor Muscle Activity in a Young Woman-A Case Report
}

\author{
Julia Konrad ${ }^{1}$, Magdalena Grygiel' \\ ${ }^{1}$ Institute of Health Sciences, University of Opole \\ Correspondence to: Julia Konrad, \\ Institute of Health Sciences, University of Opole, Katowicka 68, 45-060 Opole, Poland, \\ julia.konrad@uni.opole.pl
}

DOI: https://doi.org/10.5114/phr.2021.104599

Received: 30.12.2020 Reviewed: 23.01.2021 Accepted: 26.01.2021

\begin{abstract}
Aims: In recent years, devices for deep electromagnetic stimulation have been implemented in clinical practice. These devices generate pulses with a frequency of 1 to $50 \mathrm{~Hz}$ and magnetic induction of up to $2.5 \mathrm{~T}$. Due to the possibility of producing or an effective deep-penetrating mechanical vibration, electromagnetic stimulation is increasingly used in the physiotherapy of urogynecological disorders. This study aimed to analyse of the effect of deep electromagnetic stimulation on the bioelectrical activity of pelvic floor muscles in a young woman.
\end{abstract}

Case report: The case of a 24-year-old woman with no diagnosed stress urinary incontinence is presented. The subject underwent electromagnetic stimulation twice a week for one month (8 treatments). A single treatment lasted 15 minutes and used a magnetic induction of $1.0 \mathrm{~T}$ and a frequency that varied in each successive treatment to $50 \mathrm{~Hz}$. The bioelectrical activity of the pelvic floor muscles was measured before and after the study period. After completion of the treatment series, a decrease in muscle tone was found.

Summary: In the case of a young woman without symptoms of stress urinary incontinence, electromagnetic stimulation (with the parameters specified in the present study) was not effective for strengthening the pelvic floor muscles. Further clinical studies should be conducted to determine the appropriate management algorithm.

\section{Key words}

electromagnetic stimulation, pelvic floor muscles 


\section{Introduction}

The magnetic field is one of the more common physical agents widely used in physiotherapy. In the case of magnetic field therapy typically alternating magnetic fields with frequencies from 0.5 to $50 \mathrm{~Hz}$ and magnetic induction in the range of 0.1 to $20 \mathrm{mT}$ are used. On the other hand, in the case of magnetic field stimulation, applications are carried out at intensities from $1 \mathrm{pT}$ to $100 \mu \mathrm{T}$ and frequencies from several to several hundred thousand $\mathrm{Hz}[1]$.

In recent years, devices for deep electromagnetic stimulation, which generate impulses with frequencies from 1 to $50 \mathrm{~Hz}$ and magnetic inductions up to $2.5 \mathrm{~T}$, have been implemented in clinical practice. According to recent studies [2, $3]$, during the application, nerve and muscle fibres are stimulated and flow in the blood vessels is increased.

The most commonly used devices are highly inductive and deeply penetrating and pulsate with the help of a stimulator that allows for the local application of a precise electromagnetic field. This field penetrates the layers of clothing and the surface of the skin, and acting directly on deep tissues (the penetration reaches up to 10 $\mathrm{cm})$. This new variety of treatment is characterized by tingling and vibrating sensations felt by the patient during the therapy. Its application is also non-invasive and comfortable for the patient [3].

Due to the possibility of obtaining effective mechanical vibrations in the tissues located deeply under the skin surface, electromagnetic stimulation is increasingly used in physiotherapy of urogynecological disorders.

\section{Aims}

The objective of this study was to analyse the effect of deep electromagnetic stimulation on the bioelectrical activity of pelvic floor muscles in a young woman.

\section{Case report}

Our subject was a 24-year-old childless woman (body height of $170 \mathrm{~cm}$, body weight of $64 \mathrm{~kg}$, and BMI of $22.15 \mathrm{~kg} / \mathrm{m} 2$ ) with no diagnosed stress urinary incontinence. At first, a clinical anamnesis was performed, and it was established that the woman led a moderately active lifestyle. At the time of the study, she was a physiotherapy student doing her clinical training in hospital wards, was not on any diet, and consumed meat. The participant did not engage in regular or competitive sports, but only in recreational activitiesroller skating and some general fitness training at home-twice a week. On the occasion of physical exercise, she also performed prophylactic pelvic floor muscle training twice a day from low to high positions ( 3 series of 10 muscle contractions and relaxations consisting of $7 \mathrm{~s}$ of contraction followed by $14 \mathrm{~s}$ of relaxation).

The woman reported traveling mainly by car. No comorbidities were identified, although she had a history of periodic chronic headaches and an allergic reaction to cold, for which she took one tablet of an antihistamine drug $(10 \mathrm{mg}$ cetirizine dihydrochloride) daily. Her menstrual cycle length was 28 days, and she used oral hormonal contraception (60 $\mu$ g gestodene and $15 \mu \mathrm{g}$ ethinylestradiol daily) in a system of 24 tablets containing the active substance and 4 placebo (inactive) tablets. She had withdrawal bleeding for 4 to 5 days and reported complaints of lower abdominal pain on the first day of bleeding, rated 4 on the visual analogue scale (VAS). The woman was sexually active, having regular intercourse.

During the anamnesis, the woman did not report any noticeable changes or dysfunctions within the genitourinary system. However, she pointed out the existence of many everyday situations causing chronic psychological stress. The woman had the results of her serum cortisol level. The normal range of cortisol between 7:00 a.m. and 10:00 a.m. is $6.2 \mathrm{ug} / \mathrm{dl}$ to $19.4 \mathrm{ug} / \mathrm{dl}$, while she had a result of $25.34 \mathrm{ug} / \mathrm{dl}$. In addition, a physical examination of her body posture revealed increased lumbar lordosis and anterior pelvic tilt. 
The bioelectrical activity of the woman's pelvic floor muscles was measured using a surface electromyography (sEMG) device, NeuroTrac MyoPlus4 (Verity Medical Ltd., Tagoat, Ireland) (Figure 1). In addition, we used an endovaginal electrode Optima 3 (Sugar International, Gémenos, France) connected to the A channel of the sEMG device and a reference electrode (hypoallergenic, self-adhesive, round, 3-cm diameter) placed on the superior anterior iliac spine (a bioelectrically inactive area) (Figure 2). The measurements (before and after the completion of the project) were taken in the same room, under the same conditions, and by the same investigator to avoid any measurement errors and to enhance the reliability of the study.

For the electromagnetic stimulation procedures, we used a Salus Talent device (BTL Industries Ltd., Greeneville, USA) (Figure 3). The equipment has the following technical parameters: maximum magnetic induction $2.5 \mathrm{~T}$, maximum frequency $50 \mathrm{~Hz}$, and an applicator with a diameter of $16 \mathrm{~cm}$ placed on the patient's seat. All study procedures and desired sensations during the treatment were presented and explained to the patient prior to therapy. Deep electromagnetic stimulation was performed in a seated position (the applicator was located under the buttocks in the perineal area during the procedure and installed in the seat) in a specialized chair. The woman did not have to undress for the treatment session because electromagnetic fields penetrate everyday clothing. The therapy consisted of stimulations twice a week for a month (8 treatments). Each treatment lasted 15 minutes. During the treatment, a magnetic induction of $1.0 \mathrm{~T}$ and a frequency ranging up to $50 \mathrm{~Hz}$ during each treatment were used.

After completing the series of treatments, a reduction in muscle tension was found in all measurements (both with eyes open and closed). The results from individual muscles for the contraction phase are shown in Table 1 and for the relaxation phase in Table 2.

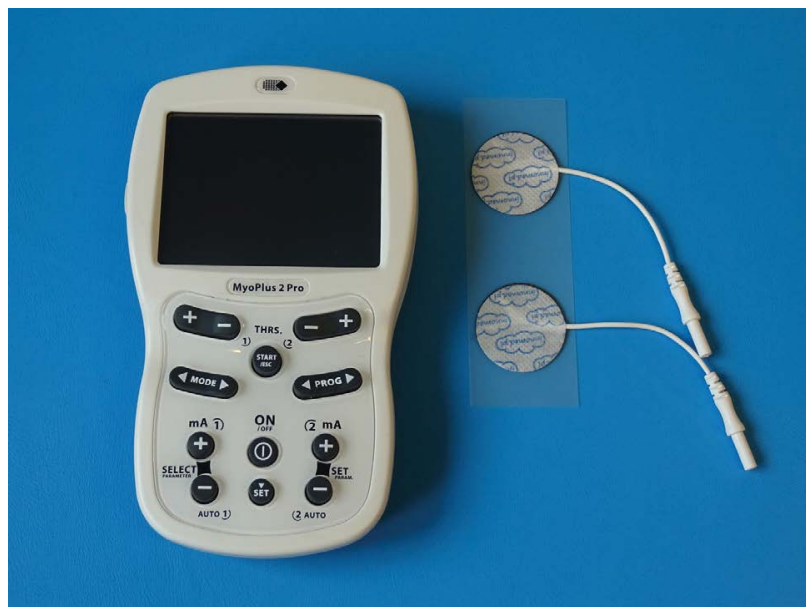

Figure 1. Surface electromyography (sEMG) device, NeuroTrac MyoPlus4 used to measure the bioelectrical activity of the woman's pelvic floor muscles.

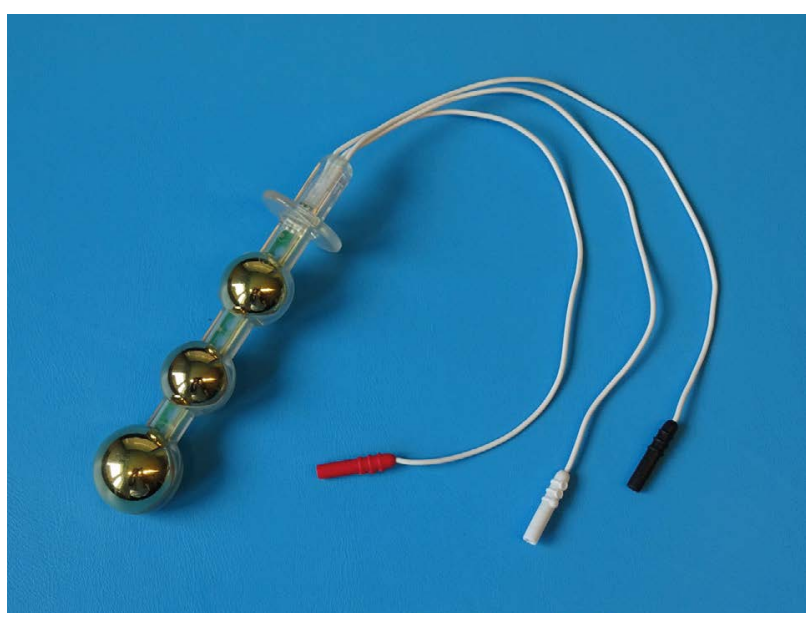

Figure 2. An endovaginal electrode Optima 3.

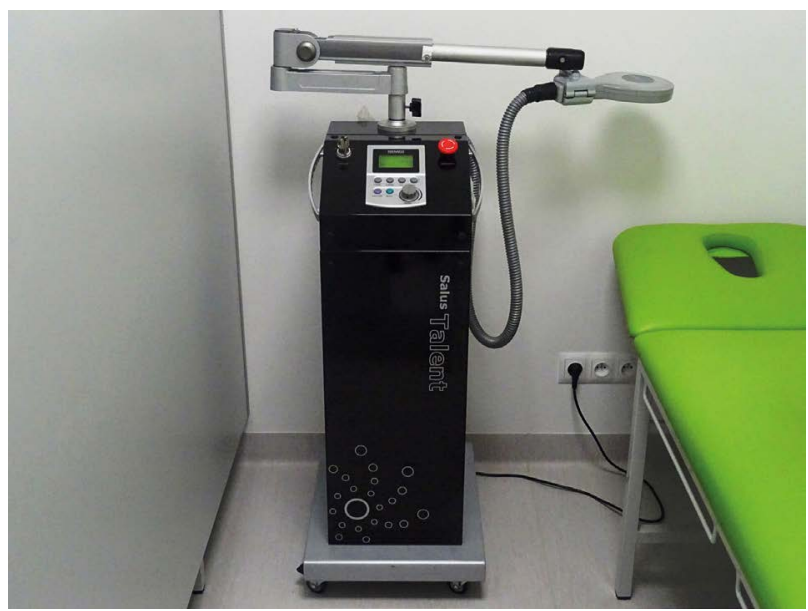

Figure 3. Salus Talent device used in electromagnetic stimulation. 
Table 1. Changes in the bioelectrical activity of pelvic floor muscles during the contraction phase under visual control before and after the treatment series.

\begin{tabular}{|c|c|c|}
\hline Muscle group tested & $\begin{array}{c}\text { Measurement under visual } \\
\text { control before a treatment series } \\
{[\mu \mathrm{V}]}\end{array}$ & $\begin{array}{c}\text { Measurement under visual } \\
\text { control after a treatment series } \\
{[\mu \mathrm{V}]}\end{array}$ \\
\hline Anterior deep muscles & 19.4 & 8.9 \\
\hline Anterior superficial muscles & 19.2 & 8.6 \\
\hline Posterior superficial muscles & 18.8 & 7.8 \\
\hline Posterior deep muscles & 17.2 & 8.4 \\
\hline
\end{tabular}

Legend: $\mu \mathrm{V}-$ microvolts.

Table 2. Changes in the bioelectrical activity of pelvic floor muscles during the relaxation phase under visual control before and after the treatment series.

\begin{tabular}{|c|c|c|}
\hline Muscle group tested & $\begin{array}{c}\text { Measurement under visual } \\
\text { control before a treatment series } \\
{[\mu \mathrm{V}]}\end{array}$ & $\begin{array}{c}\text { Measurement under visual } \\
\text { control after a treatment series } \\
{[\mu \mathrm{V}]}\end{array}$ \\
\hline Anterior deep muscles & 10.5 & 4.6 \\
\hline Anterior superficial muscles & 10.3 & 4.1 \\
\hline Posterior superficial muscles & 8.6 & 3.7 \\
\hline Posterior deep muscles & 9.3 & 3.9 \\
\hline
\end{tabular}

Legend: $\mu \mathrm{V}-$ microvolts.

\section{Discussion}

Electromagnetic stimulation is a relatively new therapeutic modality for urogynecological conditions, with only a few reports from this field in the available literature.

Ptaszkowski et al. [4] in publication from March 2020 reported results from a randomized clinical trial (RCT) in which 41 female patients with urinary incontinence (median duration of symptoms 6.5 years) were assigned to two comparison groups. In the first group $(n=21)$, the women underwent a single 20-minute electromagnetic stimulation with a high induction of $2.5 \mathrm{~T}$. The second group $(\mathrm{n}=20)$ received sham (single-blinded) treatments. The therapeutic progress was evaluated by means of SEMG of the pelvic floor muscles using an endovaginal electrode. The authors found beneficial increases in muscle tone, concluding that this procedure may be useful in the treatment of urinary incontinence.

Samuels et al. [5] in their paper from 2019 also observed a positive effect of high-intensity electromagnetic stimulation $(2.5 \mathrm{~T})$ on quality-of-life 
indicators in women with stress urinary incontinence. After a series of 6 treatments, the authors reported a reduction in symptoms of the condition in up to $81.33 \%$ of patients based on $\mathrm{qu}^{-}$ estionnaires and surveys. The project did not use sEMG recordings but only subjective research tools. These findings are supported by Silantyeva et al. [6], who found promising clinical improvement in women with stress urinary incontinence during short-term observation.

In the presented case report, unfortunately, we did not record a beneficial increase in muscle tone. At the end of the one-month programme (8 treatments), all measurements of the subject showed significant decreases in bioelectrical activity within the measured muscles. Therefore, the resulting relaxed muscle tone does not support the high effectiveness of our therapy.

To date, there are no studies in the available literature that have evaluated the effects of electromagnetic stimulation on the bioelectrical activity of pelvic floor muscles in theoretically healthy women with no history of any urogenital problems. In our case, the study participant was a young 24-year-old and otherwise healthy woman. The main intention of the therapy provided was additional muscle strengthening. The articles cited above describe the beneficial effect of this physical agent in much older women and with chronic incontinence-i.e., with significant flaccidity of muscle tone. Perhaps in cases of appropriate baseline tone of pelvic floor muscles in young women, this method is not successful in increasing muscle tone.

There are also several other factors that may have potentially affected the study results obtained. First of all, our programme used a magnetic induction of $1 \mathrm{~T}$ (an intensity of $2.5 \mathrm{~T}$ was used in the publications cited above), which seems to be ineffective in inducing significant vibration and intense pelvic floor muscle training. Unfortunately, there are no recommendations or well-established protocols regarding optimal doses and methodology, which introduces considerable latitude in the choice of treatment parameters.
It should be pointed out that the obtained results should be verified on a larger and representative population.

A 2018 preclinical study conducted by researchers from Chile and Sweden on an animal model suggests that chronic stress negatively affects the somatic system and skeletal muscles, disrupting their anabolic and catabolic balance [7]. The authors concluded that similar mechanisms may be observed clinically in humans. In turn, the authors of yet another study found that glucocorticoids strongly modulate myostatin, which is a muscle growth differentiation factor that leads to muscle atrophy [8].

Another reason for these results may be that the woman was using hormonal contraception. A 2016 systematic review and meta-analysis of 13 studies suggested that oral hormonal contraception may have an effect on pelvic floor function, although the results of RCTs were inconclusive [9]. In addition, significantly higher pelvic floor muscle bioelectrical activity during relaxation was observed in the studied women. This may be related to the anterior pelvic tilt, as studies have shown that as the anterior pelvic tilt angle increases, the bioelectrical activity of the pelvic floor muscles increases during rest and activation phases [10].

\section{Summary}

The presented case report indicates that electromagnetic stimulation (with the parameters selected for the present project) is not effective in strengthening pelvic floor muscles in a young woman without symptoms of stress urinary incontinence. However, further well-designed clinical studies should be conducted to determine an appropriate management algorithm to verify this concept. 


\section{References}

1. Markov MS. Magnetic field therapy: a review. Electromagn Biol Med. 2007; 6 (1): 1-23.

2. Rossini PM, Burke D, Chen R, Cohen LG, Daskalakis Z, Di Iorio R. et al. Non-invasive electrical and magnetic stimulation of the brain, spinal cord, roots and peripheral nerves: Basic principles and procedures for routine clinical and research application. An updated report from an I.F.C.N. Committee. Clin Neurophysiol. 2015; 126 (6): 1071-1107.

3. Yamaguchi-Sekino S, Sekino M, Ueno S. Biological effects of electromagnetic fields and recently updated safety guidelines for strong static magnetic Fields, Magn Reson Med Sci. 2011; 10 (1): 1-10.

4. Ptaszkowski K, Malkiewicz B, Zdrojowy R, Ptaszkowska L, Paprocka-Borowicz M. Assessment of the Short-Term Effects after High-Inductive Electromagnetic Stimulation of Pelvic Floor Muscles: A Randomized, Sham-Controlled Study. J Clin Med. 2020; 9 (3): 874.

5. Samuels JB, Pezzella A, Berenholz J, Alinsod R. Safety and Efficacy of a Non-Invasive High-Intensity Focused Electromagnetic Field (HIFEM) Device for Treatment of Urinary Incontinence and Enhancement of Quality of Life. Lasers Surg Med. 2019; 51: 760-766.

6. Silantyeva E, Zarkovic D, Astafeva E, Soldatskaia R, Orazov M, Belkovskaya M. et al. Academician of the Russian Academy of Sciences A Comparative Study on the Effects of High-Intensity Focused Electromagnetic Technology and Electrostimulation for the Treatment of Pelvic Floor Muscles and Urinary Incontinence in Parous Women: Analysis of Posttreatment Data. Female Pelvic Med Reconstr Surg. 2019; 25 [ahead of epub]

7. Valenzuela CA, Zuloaga R, Mercado L, Einarsdottir IE, Björnsson BT, Valdés JA. et al. Chronic stress inhibits growth and induces proteolytic mechanism through two different nonoverlapping pathways in the skeletal muscle of a teleost fish, Am J Physiol Regul Integr Comp Physiol. 2018; 314 (1): 102-113.

8. Galt NJ, McCormick SD, Froehlich JM, Biga PR. A comparative examination of cortisol effects on muscle myostatin and HSP90 gene expression in salmonids, Gen Comp Endocrinol. 2016; 237: 19-26.
9. Champaneria R, D'Andrea RM, Latthe PM. Hormonal contraception and pelvic floor function: a systematic review, Int Urogynecol J. 2016; 27 (5): 709722.

10. Lemos AQ, Brasil CA, Alvares CM, Passos JCG, Lordêlo P, Sá KN. The relation of the pelvis and the perineal function in incontinent women: a neglected subject, Neurourol Urodyn. 2018; 37 (8): 2799-2809. 